\title{
Spontaneous regression of malignant tumors: Importance of the immune system and other factors (Review)
}

\author{
SANTE BASSO RICCI ${ }^{1}$ and UGO CERCHIARI ${ }^{2}$ \\ ${ }^{1}$ Department of Radiotherapy, and ${ }^{2}$ Medical Physics Service, \\ Fondazione IRCCS Istituto Nazionale per lo Studio e la Cura dei Tumori, Milan, Italy
}

Received April 28, 2010; Accepted August 18, 2010

DOI: $10.3892 / \mathrm{ol} .2010 .176$

\begin{abstract}
It has been established that malignant tumors as well as metastases, of almost all histological types, can regress spontaneously although certain histological types regress more frequently than others. Various causes thereof include apoptosis, the immune system and particular conditions of the tumor microenvironment. The action of the genome in the regression of tumors is not clear, but some data, apart from those of apoptosis, support its involvement. The hypothesis that the immune system exhibits variations in efficacy, even to a marked extent, in determining partial or total regression of tumors appears to be plausible. Such variable efficacy may be supported by blockage of growth and the proliferation of cancer cells at the level of the tumor microenvironment, the intervention of various factors such as inhibitors of metalloproteinases and angiogenesis, and the absence or scarcity of particular proteins. The consequence of such a blockage would be a relative increase in the number of natural killer cells and other elements involved in the immune system in relation to the number of circulating cancer cells in the blood. A relative increase in the number of elements of the immune system is more effective than an absolute increase, since an absolute increase is able to stimulate, as frequently occurs for feedback in biological equilibria, inhibitor receptors that reduce the efficacy of the same elements (mainly natural killer and CD8 ${ }^{+}$ $\mathrm{T}$ cells). Such an increase in the efficacy of the immune system can lead, at least in certain cases, to the so-called spontaneous regression of malignant tumors. Clinical practice has demonstrated that metastases are less frequent in patients with renal carcinoma undergoing hemodialysis compared with patients with renal carcinoma not on hemodialysis. This finding can be interpreted, in correlation with the blockage of cancer cells in tissues, as a consequence of a partial blockage of metastatic cancer cells at the level of the dialytic membrane, with a subsequent increase in the relative efficacy of the immune system.
\end{abstract}

Correspondence to: Dr Ugo Cerchiari, Istituto Nazionale Tumori, Via Venezian 1, 20133 Milan, Italy

E-mail: ugo.cerchiari@istitutotumori.mi.it

Key words: dialytic membrane, immune system, malignant tumors, spontaneous regression

\section{Contents}

1. Introduction

2. Review of the literature and discussion

\section{Introduction}

Cases of spontaneous regression of malignant tumors are of interest since they concern a disease that is difficult to cure in spite of the many types of therapy available. The cases concern not only the primary tumor, but also metastases, single or multiple. Benign tumors can also regress, but these tumors have a clinical development that is relatively different from that of malignant tumors, and a comparison with the latter is not possible.

On the basis of data from the literature, it can be concluded that almost all types of malignant tumors can regress spontaneously, although some histological types regress more frequently than others. Moreover, when referring to cases of metastases, the regression can escape observation more often than anticipated.

It should be noted that the term spontaneous regression refers to cases not subjected to any type of therapy (surgery, radiotherapy or medical). Cases of spontaneous regression of metastases after the primary tumor had undergone local therapy (surgery or radiotherapy) were also considered. Specifically, regression of metastases has been observed independently of the evolution of the primary tumor.

Factors associated with spontaneous regression primarily include apoptosis, the immune system and conditions in the tumor microenvironment, particularly the presence of inhibitors of metalloproteinases and angiogenesis and the absence or scarcity of specific proteins.

\section{Review of the literature and discussion}

On the basis of data from the literature, certain tumor types rarely regress, whereas others, such as testicular germ cell tumors and neuroblastoma, regress with a certain frequency. This regression can be partial or total. The present review refers only to cases of clinically complete regression.

Complete regression can occur in primary tumors as well as in metastases. The process of regression in the latter 
is relatively more common, particularly when one considers the regression of one or a few metastases in a patient with numerous metastases. This eventuality is relatively more frequent and clinically identified in the metastasis of renal carcinoma.

The processes involved in the spontaneous regression of tumors are mainly related to the process of apoptosis and the activity of the immune system, as well as to conditions in the tumor microenvironment. Therefore, such processes are occasionally linked more or less directly to the oncogenic suppressors of DNA.

Among tumors that present spontaneous regression, neuroblastoma is the most studied with regards to the presence of alterations of the genome, with an oncogenic or oncosuppressor effect. Mutation of the ALK gene with an oncogenic effect, originally identified in anaplastic lymphoma and successively in lung cancer, has also been observed in neuroblastoma (1). A protein that interacts with neuronal transcription and acts as an oncogene of neuroblastoma has also been reported (2). Additionally, a transcriptional gene, a member of the kmesin protein superfamily, which induces apoptotic cell death, has been identified in a homozygously deleted region of chromosome 1p36.2 (3). Consequently, at least one oncosuppressor gene for neuroblastoma exists in conditions of normality at the level of chromosome 1p36.2 which is not dependent on the p53 gene. However, the latter remains the oncosuppressor gene most widely used for tumors, in particular for breast cancer.

Cases of spontaneous regression of thymoma of the mediastinum are relatively rare. Such tumors are also associated with myasthenia gravis, which deteriorates with regression of the tumor. Characteristic pathologic findings have not been found in these cases, nor have the causes that determine regression been identified (4). The fact that such tumors can regress upon administration of glucocorticoids suggests that hormone receptors, which act via an apoptotic mechanism, are at the fundamental cause of the regression.

Cases of spontaneous regression of testicular germ cell tumors are relatively frequent. The pathological findings of the spontaneous regression of these tumors show a distinctive constellation of findings that usually permit identification thereof (5). Among testicular germ cell tumors, only non-seminomatous and particularly choriocarcinoma are accompanied by hormonal secretion, particularly $\alpha$-fetoprotein and $\beta$-human chorionic gonadotropin. The remaining types of testicular germ cell tumors are not normally accompanied by hormonal secretions, and it is thus more difficult to consider a mechanism of apoptotic regression for the latter tumors.

Cases of spontaneous regression are also relatively frequent in melanoma and cutaneous basal cell carcinoma. Owing to the cutaneous localization, such regressions are easily identified. A mutation in the gene designated CDKN4 was found, which may contribute to the incident of melanoma (6). The immune system is most likely at the basis of the regression, since at least three antigens were located in the lymphocytes of these patients. The genes encoding these antigens have been cloned and used in vaccines of uncertain efficacy. CD4 $4^{+} \mathrm{T}$ lymphocytes and Th1 cytokines were commonly found in the body of tumors in regression (6). Moreover, melanoma cells present cytokine receptors with a high frequency.
The causes of spontaneous regression of the most studied tumors are presumably multiple, although for some of the tumors, particular causes appear to have greater importance. The majority of cases of spontaneous regression have not undergone genome testing but only laboratory analyses and examinations of the pathologic findings. The latter rarely present specific characteristics, as in the case of testicular germ cell tumors. However, extensive necrotic phenomena and infiltration of lymphocytes are prevalent. There is an increase in cytokines, such as tumor necrosis factor $\alpha$ and interleukin (IL)-18, particularly in cases of hepatocellular carcinoma (7). Given the presence of extensive necrotic phenomena, it has not been possible to ascertain whether an immune mechanism is responsible for an effective host response. Among the concomitant causes, pathologic alterations, such as arterial spasms, hemorrhage and inflammatory processes, are sometimes indicated. No reports exist in the literature of patients with eventual second tumors who have presented spontaneous tumor regression.

In the analysis of cases of the spontaneous regression of tumors, it is appropriate to consider primarily the actual entity of these cases, and in particular the metastases that regress in a still subclinical state and therefore remain undetected.

In 1998, the American Journal of Clinical Oncology published a review that analyzed a series of 217 cases of long-term relapses of breast cancer at no less than 8 years from mastectomy performed at the National Cancer Institute of Milan. Cases positive for estrogen receptors (ER+) demonstrated a statistically significant prevalence compared to negative cases (ER-). In another study published on the same subject and on the same series after a review of false-positive cases for estrogen receptors, the $\mathrm{ER}^{+}$cases represented the totality of long-term relapses. It was concluded that, following an interval of 8 years from mastectomy and without precautional therapy, recurrences appeared only in patients with $\mathrm{ER}^{+}$tumors $(8,9)$. Since the patients were not treated preventively with antiestrogens, allowing the estrogen receptors to bind with circulating estrogens and increasing cell proliferation of the breast cancer, the presence of longterm relapses could only be interpreted as the intervention of antineoplastic processes that had retarded the growth of cancer cells.

Moreover, the fact that the above cases of long-term relapses were found only in $\mathrm{ER}^{+}$breast cancer patients may be the result of relatively common spontaneous regression of the apoptosis of microscopic recurrences that occur early and are clinically not noted in these patients. Notably, these $\mathrm{ER}^{+}$breast cancer patients frequently undergo the process of apoptosis in the presence of hormone receptors. In contrast, such cases of regression are exceptional in ER patients, given the absence in these patients of the apoptotic process. Therefore, an eventual long-term relapse 8 years after surgery in $\mathrm{ER}^{-}$patients is almost always preceded by a first recurrence already noted clinically, whereas in $\mathrm{ER}^{+}$patients a relapse 8 years after surgery often appears as a first relapse. It should also be noted that recurrences at a subclinical state have a greater probability of complete regression, compared to larger recurrences, since in the latter there is a smaller possibility of verifying an apoptotic process in all of the cells when compared to recurrences consisting of few cells. 
Other factors can act favorably on the prognosis of $\mathrm{ER}^{+}$ breast cancer by increasing the incidence of long-term relapses. Estrogen receptors regulate the expression of insulin-like growth receptor factor 1 by increasing its favorable effect and reducing BCL2, an antiapoptotic protein, without affecting the expression of Bax, a pro-apoptotic protein (10-12). However, the presence of the epidermal growth factor can overcome the action of estrogen receptors and unfavorably affect neoplastic development (13).

The majority of cases of tumors with long-term relapses concern cancer of the breast, uterus (14), thyroid (15), and prostate (16), i.e., tumors considered hormone-dependent. These tumors express hormone receptors more often than other tumors and undergo the process of apoptosis.

The activity of the p53 gene, which is the most known and active in the process of apoptosis, depends on a transcriptiondependent or -independent mechanism (17). Moreover, the p53 gene presents alterations and mutations that inhibit its function in more than $50 \%$ of breast cancer patients (17). This is one of the most likely causes of the frequent inability of apoptosis to yield complete clinical results. Nevertheless, the positive prognostic action of receptors linked to estrogen is mainly related to stimulation of the p53 gene by a transcription-dependent mechanism.

A transcription-independent mechanism is also possible, and a variable number of p53 extragenome molecules, independent of the transcription mechanism, are present in the nucleus. The molecules may be increased to a high level if required. Such molecules are associated with an inactivating protein complex, and rupture of this complex is possible, with the release of active p53.

It can be concluded that the process of apoptosis definitely plays an important role in cases of spontaneous tumor regression and is linked to a high percentage of cases with the presence of hormone receptors. However, apoptosis is carried out through an internal cell process, poorly modifiable from the outside.

In the elimination of cancer cells, it is possible that damage caused to cancer cells by various elements of the immune system during the clinical evolution of the tumor, from carcinogenesis to metastatic dissemination and to colonization in various regions of the organism, is cumulative. Such cumulative damage is favored by the fact that, when natural killer (NK) cells, T leukocytes and fagocytosing leukocytes infiltrate a tumor mass, they produce the same type of damage to cancer cells. Such damage includes cytolysis, which also characterizes apoptosis, even when the latter is programmed within the cell and is not the result of elements of the immune system. Additionally, certain apoptotic processes cause only partial damage, without necessarily resulting in cell death. In this case, such damage accumulates with further damage which is evident in cancer cells. Such an accumulation of damage is lethal to a certain number of cancer cells and reduces the vitality and aggressivity of the remaining cells.

Regarding the importance of the immune system in the presence of malignant tumors, it should be noted that innate immunity is largely predominant over adaptive immunity. Eventual antibodies present are almost always insufficient to determine clinically significant results. However, it is likely that alterations in cell proteins in the cancer cells by various elements of the immune system lead to the formation of macromolecular complexes with the potentiality of antigens that escape the mechanisms of self defense of the major histocompatibility complex (MHC) that may induce antibodies. In the course of apoptosis, particular antigens can be formed that cause antibodies (18). Among cases of spontaneous regression of malignant tumors, there are cases in which the importance of eventual antibodies can be excluded, since spontaneous regressions have been found in tumors of the central nervous system (19) where the presence of antibodies is excluded given the presence of the blood-brain barrier.

NK cells are important elements of the immune system. These cells play an important role in the protection against transplantable tumors. Such antitumor effects can be intensified by the presence of cytokines such as IL-8, -12, -15 and interferon $\alpha / \beta$ (20). Although a number of the results have been obtained with experimental studies on animals, it has been ascertained that such cytokines possess the capacity to compel human-activated NK cells to secrete other cytokines and to kill tumor cells. Nevertheless, it should be noted that to achieve a prominent clinical result, the cytokines that stimulate NK cells against cancer cells must be introduced externally in a sufficient quantity. It should also be noted that these cytokines are toxic.

The activity of NK cells is related to the presence of activating and inhibitory NK receptors. T cells may also have the same types of receptors. Since such receptors play significant roles in cases of spontaneous tumor regression, further investigation of this subject is warranted.

The majority of activating receptors are associated with the immunoreceptor tyrosine-based activating motif (ITAM) (21) and MHC. The final effect is the induction of gene transcription with the activation of NK cells (21).

Other activating NK receptors functioning through ITAMbearing adaptor proteins include CD 16 (22) and natural cytotoxic receptors.

Currently, at least two families of inhibitor receptors of NK cells have been identified in humans. The first family is represented by killer cell immunoglobulin-like receptors (KIR). Most KIR molecules possess two immunoreceptor tyrosine-based inhibitory motifs (reviewed in ref. 20). The second family of inhibitors consists of disulfide-bound heterodimers of CD94 and NKG2A (23). These receptor complexes are expressed on approximately half of the NK cells and on a subset of $\mathrm{CD}^{+} \mathrm{T}$ cells and allow recruitment of tyrosine phosphatases to exert an inhibitory effect. In this case, $\mathrm{NK}$ and $\mathrm{CD}^{+} \mathrm{T}$ cells monitor the general expression of MHC class 1 molecules (24).

Based on the above findings, it may be concluded that, relative to the action of activating and inhibitory receptors of NK cells, a marked difference among individuals is possible. Given the variety of receptor types, the presence of these cells in different quantities, the diverse efficacy of even the same type, the possibility of an opposite action and the variability of a relation with $\mathrm{MHC}$, a marked difference in the clinical effects from individual to individual is feasible.

$\mathrm{CD}^{+} \mathrm{T}$ cells are also considered to be markedly important, particularly after infiltration in tumor masses involving their cytolytic action. 
In addition to apoptosis and the immune system, the conditions of the tumor microenvironment are also important in hindering the growth of a tumor. The presence of inhibitors of metalloproteinases, the presence of anti-angiogenic factors, the scarcity or absence of well-characterized epithelial cadherin proteins (25), as well as the scarcity or absence of factors of the large integrin family (26) and of adenosine (27) inhibit growth of the primary tumor and metastases. Occasionally, incidental events, such as ischemia, hemorrhage and inflammatory processes, can also be determinants. Of note is that metastatic cells that reach tissues may present cytolytic damage caused by elements of the immune system and are therefore less aggressive. Moreover, metastatic cancer cells recover from damage less readily than normal cells.

The passage of metastatic cancer cells into tissues and the lack of growth and proliferation of a number of them due to opposing factors of the microenvironment reduce the number of cancer cells circulating in the blood and indirectly increase the possibility of immune system defense. Moreover, metastatic cells that eventually reach the lymph nodes (which are highly active sites of the immune system), in addition to being partially blocked, markedly stimulate the immune system. Cases of spontaneous regression of lymph node metastases of carcinoma have been reported (28).

However, factors that can lead to the blockage of growth of metastatic cells in tissues are always opposed by factors that have contrasting action and that often annul the blocking action. Subsequently, the fact that cases of spontaneous tumor regression are decidedly rare can be explained.

Apoptosis, the immune system with its activating receptors of NK cells, and the presence or absence of various factors of the microenvironment can explain, particularly when all these factors are coadjuvant, cases of spontaneous regression, total or partial, of the primary tumor and of its metastases.

Identifying the most stimulating factors involved in the therapy and regression of tumors is crucial. A hypothesis, aforementioned in generic terms, is to be formulated in detail. The previously mentioned factors that oppose the growth and proliferation of cancer cells can result in the blockage of development of oncologic disease and a reduction in the number of active cancer cells. The indirect effect is to determine an increase, not absolute but relative, in the elements of the immune system, compared to cancer cells. Therefore, the efficacy of the immune system against cancer cells increases, particularly when the primary tumor has undergone surgery. Cases of regression of metastases after surgery of the primitive tumor, in particular of renal carcinoma, have been identified with relative frequency $(29,30)$.

It should be noted that an increased absolute number of NK cells has less efficacy against cancer cells than an increase in NK cells relative to the number of cancer cells. An absolute increase in NK cells stimulates inhibitory receptors, as frequently occurs for feedback in biological equilibria, which reduces the efficacy of NK cells, resulting in unfavorable prognosis.

Moreover, in the presence of a relative increase in NK cells, and when the activity of the activating receptors is more effective than that of inhibitory receptors, it is likely that the cumulative cytolytic damage overcomes the threshold of resis- tance of the cancer cells to the point where death of the latter is determined.

Based on the above facts, in order to strengthen the innate immunity against cancer cells, the most effective therapeutic treatment is a relative increase in the elements of the immune system along with cancer cell blockage in the tissues.

In brief, it is possible to conclude that the most effective action involved in spontaneous tumor regression is by the immune system and that the strongest stimulation of the latter is presumably by the eventual blockage in the tissues, albeit partial, of metastatic cancer cells. Apoptosis is a factor that can be important, but it is part of an internal process since the cell cannot be externally modified.

Although the blockage of cancer cells in the tissues, apoptosis and the effects of the immune system can lead to total regression of malignant tumors in a few cases, such a blockage results in only a partially favorable outcome in the clinical evolution of the oncologic disease. Since the most favorable effect is an increase in immune system elements relative to cancer cells, achieving this relative increase warrants investigation.

Current clinical practice provides possible proof of the hypothesis stated above. A recent study showed a marked reduction in the incidence of metastases, after postmortem examination, in patients with renal carcinoma on hemodialysis, compared to patients with renal carcinoma not on hemodialysis. In patients on hemodialysis, it is probable that there is a block of metastatic cancer cells at the level of the dialytic membrane (31). In this case, the blockage of cancer cells does not occur in a biological sense as in the case of cancer cells in tissues, but in a physical sense (blockade), albeit the effect is equivalent. The blockage by the dialytic membrane reduces the number of cancer cells circulating in the blood, and the immune system elements would thus have to oppose a smaller number of cancer cells. The cancer cells blocked by the dialytic membrane are then eliminated by the periodic intervention of the extracorporeal circulation.

Even when aforementioned factors, in part, block the growth and proliferation of cancer cells in the tissues of the organism, the cells remain present in the organism and continue to exert their stimulating action on the immune system. Cytokines able to stimulate NK cells may also be present in the circulating blood, contributing to the defense of the organism. Such cytokines are present only in a limited quantity. Nevertheless, despite their toxicity cytokines should be externally introduced to be effective.

Treatment with most chemotherapy agents reduces the proliferation of cancer cells, reducing their number, and increasing the number of cells relative to the immune system. However, the majority of agents exert a negative action on the immune system, thereby reducing its efficacy. In contrast, the blockage of cancer cells by a dialytic membrane as well as blockage of these cells and their proliferation by tissues of the body, and the elimination from the blood circulation of a certain number of cancer cells, may positively stimulate the immune system without negative effects. In conclusion, blockage of the growth and proliferation of cancer cells in tissues of the organism, as well as the presence of the dialytic membrane, can play a significantly positive role in the development and spontaneous and total regression of malignant tumors. 


\section{References}

1. Chen Y, Takita J, Choi YL, et al: Oncogenic mutations of ALK kinase in neuroblastoma. Nature 455: 971-974, 2008.

2. Aoyama M, Ozaki T, Inuzuka H, et al: LMO3 interacts with neuronal transcription factor HEN2 and acts as an oncogene in neuroblastoma. Cancer Res 65: 4587-4597, 2005.

3. Munirajan AK, Ando K, Mukai A, et al: KIF1B(beta) functions as a haploinsufficient tumor suppressor gene mapped to chromosome 1p36.2 by inducing apoptotic cell death. J Biol Chem 283: 24426-24434, 2008.

4. Huang TW, Chen YL, Chen JC, Tsai WC, Chang H and Lee SC: Spontaneous regression of a mediastinal thymoma. J Thorac Cardiovasc Surg 137: 1277-1278, 2009.

5. Balzer BL and Ulbright TM: Spontaneous regression of testicular germ cell tumors. An analysis of 42 cases. Am J Surg Pathol 30: 858-865, 2006.

6. Printz C: Spontaneous regression of melanoma may offer insight into cancer immunology. J Natl Cancer Inst 93: 1047-1048, 2001.

7. Li AJ, Wu MC, Cong WM, Shen F and Yi B: Spontaneous complete necrosis of hepatocellular carcinoma: a case report. Hepatobiliary Pancreat Dis Int 2: 152-154, 2003.

8. Basso Ricci S, Coradini D, Di Fronzo G, Bartoli C and Villa S: Estrogen receptor status of patients subjected to mastectomy for breast cancer with a disease-free interval of no less than 8 years. Am J Clin Oncol 21: 250-252, 1998.

9. Basso Ricci S: Letter to the editor. Med Hypothesis 64: 428-431, 2005.

10. Reed JC: Balancing cell life and death. Bax, apoptosis, and breast cancer. J Clin Invest 97: 2403-2404, 1996.

11. Teixeira C, Reed JC and Pratt MAC: Estrogen promotes chemotherapeutic drug resistance by a mechanism involving $\mathrm{Bcl}-2$ proto-oncogene expression in human breast cancer cells. Cancer Res 55: 3902-3912, 1995.

12. Wang TTY and Phang JM: Effects of estrogen on apoptosis pathways in human breast cancer cell line MCF-7. Cancer Res 55: 2487-2489, 1995.

13. Dikinson RB and Lipman ME: Growth regulation of norma and malignant breast epithelium. In: The Breast: Comprehensive Management of Benign and Malignant Diseases. 2nd edition. Vol. 1. Bland K and Copeland EM III (eds). WB Saunders, Philadelphia, p518, 1998.

14. Lederman GS, Niloff JM, Redline R and Rosen EM: Late recurrence in endometrial carcinoma. Cancer 59: 825-828, 1987.

15. Tubiana N, Schlumberger M, Rougier P, et al: Long-term results and prognostic factors in patients with differentiated thyroid carcinoma. Cancer 55: 794-804, 1985.

16. Van den Ouden D, Hop WC and Schröder FH: Progression in and survival of patients with locally advanced prostate cancer (T3) treated with radical prostatectomy as monotherapy. J Urol 160: 1392-1397, 1998.
17. Wang XW: Role of $\mathrm{p} 53$ and apoptosis in carcinogenesis. Anticancer Res 19: 4759-4771, 1999.

18. Navratil JS, Liu CC and Ahearn JM: Apoptosis and autoimmunity. Immunol Res 36: 3-12, 2006.

19. Yamashita Y, Kumabe T, Shimizu H, Ezura H and Tominaga T: Spontaneous regression of a primary cerebral tumor following vasospasm caused by subarachnoid hemorrhage due to rupture of an intracranial aneurism. A case report. Neurol Med Chir 4: 187-190, 2004.

20. Wu J and Lanier LL: Natural killer cells and cancer. Adv Cancer Res 90: 127-156, 2003.

21. Lanier LL, Corliss BC, Wu J, Leong C and Phillips JH: Immunoreceptor DAP12 bearing a tyrosine-based activation motif is involved in activating NK cells. Nature 391: 703-707, 1998.

22. Lanier LL, Yu G and Phillips JH: Co-association of CD3 zeta with a receptor (CD16) for IgG Fc on human natural killer cells. Nature 142: 803-805, 1989.

23. Brooks AG, Posch PE, Scorzelli CT, Borrego F and Coligan JE: NKG2A complexed with CD94 defines a novel inhibitory natural kill cell receptor. J Exp Med 185: 795-800, 1997.

24. Lanier LL: Natural killer cell receptor signaling. Curr Opin Immunol 15: 308-314, 2003.

25. Munshi HG and Stack MS: Reciprocal interaction between adhesion receptor signaling and MMP regulation. Cancer Metastasis Rev 25: 45-56, 2006.

26. Hood JD and Cheresh DA: Role of integrins in cell invasion and migration. Natl Rev Cancer 2: 91-100, 2002.

27. Hoskin DH, Mader JS, Furlong SJ, Conrad DM and Blay J: Inhibition of T-cell and natural killer cell function by adenosine and its contribution to immune evasion by tumor cells (Review). Int J Oncol 18: 527-535, 2008.

28. Kurita M, Hirano K, Ebihara S, et al: Spontaneous regression of cervical lymph node metastasis in patients with mesopharyngeal squamous cell carcinoma of the tongue: possible association between apoptosis and tumor regression. Int J Clin Oncol 12: 448-454, 2007.

29. Thoroddsen A, Geirsson G, Agnarsson BA and Magnusson K: Spontaneous regression of pleural metastases after nephrectomy for renal cell carcinoma. Scand J Urol Nephrol 5: 396-398, 2002.

30. DeWeerd JH, Hawthorne J and Adson MA: Regression of renal cell hepatic metastases following removal of primary lesions. J Urol 117: 790-792, 1977.

31. Basso Ricci S: Dialysis membrane and metastatic cancer cells. Clin Nephrol 68: 354-356, 2007. 